\title{
Observations of GRBs with the Infrared Space Observatory ${ }^{\star}$
}

\author{
L. Hanlon ${ }^{1}$, L. Metcalfe ${ }^{2}$, M. Delaney ${ }^{1,11}$, R. Laureijs ${ }^{2}$, B. McBreen ${ }^{1}$, N. Smith ${ }^{3}$, B. Altieri ${ }^{2}$, A. Castro-Tirado ${ }^{4,12}$,

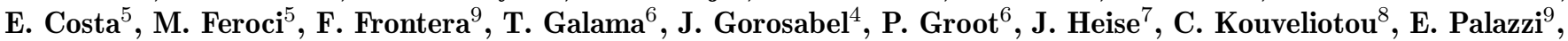 \\ J. van Paradijs ${ }^{7,10}$, L. Piro ${ }^{5}$, and M. Kessler ${ }^{2}$ \\ 1 Department of Experimental Physics, University College Dublin, Belfield, Stillorgan Road, Dublin 4, Ireland \\ 2 ISO Science Operations Centre, Astrophysics Division, Space Science Department of ESA, Villafranca, Spain \\ 3 Cork Institute of Technology, Cork, Ireland \\ ${ }^{4}$ LAEFF, Villafranca del Castillo, P.O. Box 50727, 28080 Madrid, Spain \\ ${ }^{5}$ Istituto Astrofisica Spaziale, CNR, 00133 Roma, Italy \\ 6 Astronomical Institute "Anton Pannekoek", University of Amsterdam, The Netherlands \\ 7 SRON Utrecht, The Netherlands \\ 8 USRA at NASA/MSFC, Huntsville AL, U.S.A. \\ 9 ITESRE-CNR, Bologna, Italy \\ 10 Physics Department, University of Alabama, Huntsville AL, U.S.A. \\ 11 Stockholm Observatory, SE-133 36 Saltsjöbaden, Sweden \\ 12 IAA-CSIC, P.O. Box 03004, 18080 Granada, Spain
}

Received December 18, 1998; accepted March 10, 1999

\begin{abstract}
We present results from the Target of Opportunity (TOO) program to observe GRB locations with the CAM and PHOT instruments aboard the Infrared Space Observatory (ISO). Four BeppoSAX GRB error circles were observed by ISO on timescales ranging from days to months after the GRB events. These observations represent the first prompt GRB counterpart searches at far-infrared wavelengths. A list of observations made in this TOO program, along with results of CAM follow-up observations of GRB 970508, are presented. A marginal CAM $(12 \mu \mathrm{m})$ detection of a source, consistent with the position of the optical transient of GRB 970508, is reported.
\end{abstract}

Key words: gamma-ray bursts

\section{Introduction}

The recent discoveries of fading multiwavelength afterglows (Costa et al. 1997; van Paradijs et al. 1997; Frail et al. 1997) to a number of $\gamma$-ray bursts (GRBs) have been precipitated by the accurate and prompt localisation capability of the two Wide Field Cameras (WFC) aboard the Italian-Dutch BeppoSAX X-ray satellite (Boella et al. 1997). The shrinking of GRB error

\footnotetext{
* ISO is an ESA project with instruments funded by ESA Member States (especially the PI countries: France, Germany, the Netherlands and the United Kingdom) and with the participation of ISAS and NASA.

Correspondence to: lhanlon@bermuda.ucd.ie
}

circles by BeppoSAX also made it feasible to study the content of these regions at far-infrared wavelengths with the European Space Agency's Infrared Space Observatory (ISO) which flew between November 1995 and April 1998 (Kessler et al. 1996).

A search for quiescent far-infrared emission from 23 well-localised GRBs using the IRAS faint source catalogue identified no quiescent counterparts (Schaefer et al. 1987). Compared to IRAS, ISO had broader wavelength coverage, better spatial resolution, greater sensitivity and a longer lifetime. ISO also had the added bonus that its operational lifetime serendipitously overlapped with that of BeppoSAX. In early 1997 a TOO program was awarded to make rapid follow-up observations of welllocalised BeppoSAX GRBs with ISO. The objectives of the program were to observe infrared afterglow emission from GRBs for the first time and to place constraints on the underlying hosts which may be actively star-forming regions (Bloom et al. 1998; Paczynski 1998).

\section{Observations}

Due to strong constraints on the allowed pointing directions of the satellite, only 2 out of the 12 well-localised GRBs that were detected between the beginning of the TOO program (February 1997) and the end of the ISO mission (April 1998) were immediately visible with ISO. An ISO follow-up observation of one (GRB 970402) was made within 55 hours (Castro-Tirado et al. 1998). However the second candidate (GRB 970828) was not observed due to unavoidable scheduling delays and the absence of an optical transient (OT). A further 3 


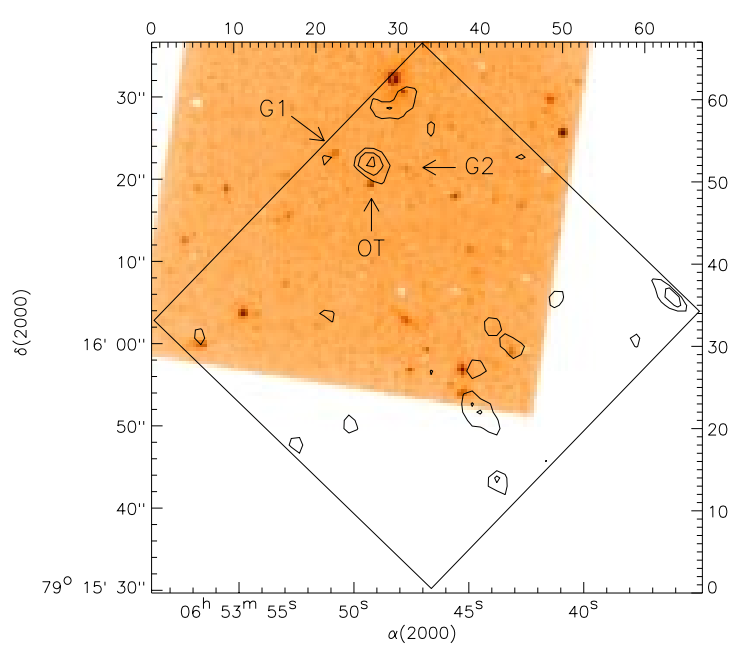

Fig. 1. The central $45^{\prime \prime} \times 45^{\prime \prime}$ region of the CAM LW10 mosaic image taken on May 24 overlaid with the HST STIS image from June 2. The images have been rotated to have North up and East left. RA and Dec scaling and LW10 pixel scaling are shown on the axes. The white "divets" are due to a flatfielding error in the STIS standard processing pipeline. The contour levels are: 2,3 and $4 \mu \mathrm{Jy} / \operatorname{arcsec}^{2}$

of the 12 GRB locations only became visible at later times. Limited viewing windows of the GRB 970508 and GRB 980329 locations opened after several days, but there was a delay of 7 months before the location of GRB 970228 could be observed. Further details of the observations and analysis methods are presented elsewhere (Delaney et al. 1999). Results on GRB 980329 and GRB 970228 are in preparation (Groot et al. 1999; Metcalfe et al. 1999).

\section{Observations of GRB 970508 with CAM}

GRB 970508 was observed on May 21 and May 24 1997 (Fig. 1) with CAM's LW10 filter $(8-15 \mu \mathrm{m}$, reference wavelength $12 \mu \mathrm{m}$, assuming a $1 / \lambda$ spectrum). Observations made with the PHOT instrument will be presented elsewhere (Hanlon et al. 1999). The most significant ( $\sim 3 \sigma$ above background) source which appears in this, the deepest region of the May 24 mosaic, is coincident with the OT associated with GRB 970508. The astrometric pointing accuracy of ISO $\left(\sim 5^{\prime \prime}\right)$ has been improved by matching several sources in the combined May 21 and May 24 full-field mosaics with optical sources. The flux of the source is $\sim 60 \mu \mathrm{Jy}$. The overall photometric error, including relative and absolute calibration uncertainties, is approximately 50\%. From Fig. 1 it can be seen that the source is not coincident with either of the faint nearby galaxies G1 and G2 (Pian et al. 1998).

The source was not detected in the May 21 observation. The flux on May 21 can be inferred by extrapolating the observed flux on May 24, assuming a decay proportional to $t^{-1.1}$ days, as was seen in the $R$ band (Galama et al. 1998a; Castro-Tirado et al. 1998). The $12 \mu \mathrm{m}$ flux on May 21, extrapolated back from May 24 is $79 \pm 35 \mu \mathrm{Jy}$, consistent with the measured $3 \sigma$ upper limit of $60 \mu \mathrm{Jy}$. We therefore cannot rule out the possibility that this is a constant source, rather than transient emission. However, the emission detected at $12 \mu \mathrm{m}$ on May 24 is consistent with the extrapolated optical to radio spectrum which has been modelled as synchrotron radiation from a population of relativistic electrons, a significant fraction of which is cooled rapidly (Galama et al. 1998b; Sari et al. 1998).

\section{Conclusions}

The ISO observations of GRBs place unique limits on the level of infrared emission in the weeks and months following the bursts. Emission at $12 \mu \mathrm{m}$ has been marginally detected from GRB 970508 and further analysis of this and other other GRBs may lead to more new detections of both transient emission and possible host galaxy signatures.

Acknowledgements. The ISOCAM data presented in this paper was analysed using "CIA", a joint development by the ESA Astrophysics Division and the ISOCAM Consortium. The ISOCAM Consortium is led by the ISOCAM PI, C. Cesarsky, Direction des Sciences de la Matiere, C.E.A., France.

\section{References}

Bloom J., Djorgovski S., Kulkarni S., Frail D., 1998, ApJ 507, L25

Boella G., Butler R., et al., 1997, A\&AS 122, 299

Castro-Tirado A., Metcalfe L., Laureijs R., et al., 1998, A\&A 330,14

Castro-Tirado A., Gorosabel J., Ben'itez N., et al., 1998, Sci 279,1011

Costa E., Frontera F., Heise J., et al., 1997, Nat 387, 783

Delaney M., Hanlon L., Metcalfe L., et al., 1999, in The Universe as seen by ISO

Frail D., Kulkarni S., Nicastro L., et al., 1997, Nat 389, 261

Galama T., Groot P., van Paradijs J., et al., 1998a, ApJ 497, L13

Galama T., Wijers R., Bremer M., et al., 1998b, ApJ 500, L101

Groot P., et al., 1999 (in preparation)

Hanlon L., et al., 1999 (in preparation)

Kessler M., Steinz J., Anderegg M., et al., 1996, A\&A 315, L27

Metcalfe L., et al., 1999 (in preparation)

Paczynski B., 1998, ApJ 494, 45

Pian E., Fruchter A., Bergeron L., et al., 1998, ApJ 492, L103

Sari R., Piran T., Narayan R., 1998, ApJ 497, 17

Schaefer B., Cline T., Desai U., et al., 1987, ApJ 313, 226

van Paradijs J., Groot P., Galama T., et al., 1997, Nat 386, 686 